\title{
Constraints on the dipole photon strength for the odd uranium isotopes
}

J. Moreno-Soto, ${ }^{1, *}$ S. Valenta, ${ }^{2, \dagger}$ E. Berthoumieux,${ }^{1}$ A. Chebboubi,${ }^{3}$ M. Diakaki, ${ }^{3}$ W. Dridi, ${ }^{4}$ E. Dupont, ${ }^{1}$ F. Gunsing,${ }^{1,+}$ M. Krtička, ${ }^{2}$ O. Litaize, ${ }^{3}$ O. Serot,${ }^{3}$ O. Aberle,${ }^{5}$ V. Alcayne, ${ }^{6}$ S. Amaducci, ${ }^{7}$ J. Andrzejewski, ${ }^{8}$ L. Audouin, ${ }^{9}$ V. Bécares, ${ }^{6}$ V. Babiano-Suarez ${ }^{10}$ M. Bacak,${ }^{5,11,1}$ M. Barbagallo, ${ }^{5,12}$ Th. Benedikt, ${ }^{13}$ S. Bennett, ${ }^{14}$ J. Billowes, ${ }^{14}$ D. Bosnar, ${ }^{15}$ A. Brown,${ }^{16}$ M. Busso, ${ }^{17,18}$ M. Caamaño, ${ }^{19}$ L. Caballero-Ontanaya, ${ }^{10}$ F. Calviño,${ }^{20}$ M. Calviani, ${ }^{5}$ D. Cano-Ott,${ }^{6}$ A. Casanovas, ${ }^{20}$ F. Cerutti, ${ }^{5}$ E. Chiaveri, ${ }^{5,14}$ N. Colonna, ${ }^{12}$ G. Cortés, ${ }^{20}$ M. A. Cortés-Giraldo ${ }^{21}$ L. Cosentino, ${ }^{7}$ S. Cristallo, ${ }^{17,22}$ L. A. Damone, ${ }^{12,23}$ P. J. Davies, ${ }^{14}$ M. Dietz, ${ }^{24}$ C. Domingo-Pardo,${ }^{10}$ R. Dressler, ${ }^{25}$ Q. Ducasse, ${ }^{26}$ I. Durán, ${ }^{19}$ Z. Eleme, ${ }^{27}$

B. Fernández-Domínguez, ${ }^{19}$ A. Ferrari, ${ }^{5}$ P. Finocchiaro, ${ }^{7}$ V. Furman, ${ }^{28}$ K. Göbel, ${ }^{13}$ A. Gawlik-Ramięga, ${ }^{8}$ S. Gilardoni, ${ }^{5}$ I. F. Gonçalves ${ }^{29}$ E. González-Romero, ${ }^{6}$ C. Guerrero, ${ }^{21}$ S. Heinitz ${ }^{25}$ J. Heyse,,${ }^{30}$ D. G. Jenkins, ${ }^{16}$ A. Junghans,${ }^{31}$ F. Käppeler, ${ }^{32,8}$ Y. Kadi,${ }^{5}$ A. Kimura ${ }^{33}$ I. Knapová, ${ }^{2}$ M. Kokkoris, ${ }^{34}$ Y. Kopatch, ${ }^{28}$ D. Kurtulgil, ${ }^{13}$ I. Ladarescu,,${ }^{10}$ C. Lampoudis,${ }^{35}$ C. Lederer-Woods, ${ }^{24}$ S. J. Lonsdale, ${ }^{24}$ D. Macina, ${ }^{5}$ A. Manna,${ }^{36,37}$ T. Martínez, ${ }^{6}$ A. Masi, ${ }^{5}$ C. Massimi, ${ }^{36,37}$ P. Mastinu, ${ }^{38}$ M. Mastromarco, ${ }^{5}$ E. A. Maugeri, ${ }^{25}$ A. Mazzone, ${ }^{12,39}$ E. Mendoza, ${ }^{6}$ A. Mengoni, ${ }^{40}$ V. Michalopoulou, ${ }^{5,34}$ P. M. Milazzo, ${ }^{41}$ F. Mingrone, ${ }^{5}$ A. Musumarra,${ }^{7,42}$ A. Negret,${ }^{43}$ R. Nolte, ${ }^{26}$ F. Ogállar, ${ }^{44}$ A. Oprea,${ }^{43}$ N. Patronis,${ }^{27}$ A. Pavlik, ${ }^{45}$ J. Perkowski, ${ }^{8}$ L. Piersanti, ${ }^{17,22}$ C. Petrone,${ }^{43}$ E. Pirovano, ${ }^{26}$ I. Porras,${ }^{44}$ J. Praena,${ }^{44}$ J. M. Quesada,${ }^{21}$ D. Ramos-Doval,${ }^{9}$ T. Rauscher ${ }^{46,47}$ R. Reifarth, ${ }^{13}$ D. Rochman, ${ }^{25}$ M. Sabaté-Gilarte, ${ }^{21,5}$ A. Saxena,${ }^{48}$ P. Schillebeeckx, ${ }^{30}$ D. Schumann, ${ }^{25}$ A. Sekhar, ${ }^{14}$ A. G. Smith, ${ }^{14}$ N. V. Sosnin, ${ }^{14}$ P. Sprung, ${ }^{25}$ A. Stamatopoulos,${ }^{34}$ G. Tagliente, ${ }^{12}$ J. L. Tain, ${ }^{10}$

A. Tarifeño-Saldivia, ${ }^{20}$ L. Tassan-Got, ${ }^{5,34,9}$ P. Torres-Sánchez,${ }^{44}$ A. Tsinganis, ${ }^{5}$ J. Ulrich, ${ }^{25}$ S. Urlass, ${ }^{31,5}$ G. Vannini, ${ }^{36,37}$ V. Variale, ${ }^{12}$ P. Vaz,${ }^{29}$ A. Ventura, ${ }^{36}$ D. Vescovi, ${ }^{17}$ V. Vlachoudis, ${ }^{5}$ R. Vlastou, ${ }^{34}$ A. Wallner, ${ }^{49}$ P. J. Woods, ${ }^{24}$ T. Wright, ${ }^{14}$ and P. Žugec ${ }^{15, \pi}$

(n_TOF Collaboration)

${ }^{1}$ CEA Irfu, Université Paris-Saclay, F-91191 Gif-sur-Yvette, France

${ }^{2}$ Faculty of Mathematics and Physics, Charles University, Prague, Czech Republic

${ }^{3}$ CEA Cadarache, DES/DER/SPRC, F-13108 Saint-Paul-lez-Durance, France

${ }^{4}$ Laboratory on Energy and Matter for Nuclear Sciences Development (LR16CNSTN02), Technopark Sidi Thabet, 2020 Ariana, Tunisia

${ }^{5}$ European Organization for Nuclear Research (CERN), Geneva, Switzerland

${ }^{6}$ Centro de Investigaciones Energéticas Medioambientales y Tecnológicas (CIEMAT), Madrid, Spain

${ }^{7}$ INFN Laboratori Nazionali del Sud, Catania, Italy

${ }^{8}$ University of Lodz, Lodz, Poland

${ }^{9}$ Institut de Physique Nucléaire, CNRS-IN2P3, Université Paris-Sud, Université Paris-Saclay, F-91406 Orsay Cedex, France

${ }^{10}$ Instituto de Física Corpuscular, CSIC - Universidad de Valencia, Valencia, Spain

${ }^{11}$ Technische Universität Wien, Austria

${ }^{12}$ Istituto Nazionale di Fisica Nucleare, Sezione di Bari, Bari, Italy

${ }^{13}$ Goethe University, Frankfurt, Germany

${ }^{14}$ University of Manchester, Manchester, United Kingdom

${ }^{15}$ Department of Physics, Faculty of Science, University of Zagreb, Zagreb, Croatia

${ }^{16}$ University of York, York, United Kingdom

${ }^{17}$ Istituto Nazionale di Fisica Nucleare, Sezione di Perugia, Perugia, Italy

${ }^{18}$ Dipartimento di Fisica e Geologia, Università di Perugia, Perugia, Italy

${ }^{19}$ University of Santiago de Compostela, Santiago de Compostela, Spain

${ }^{20}$ Universitat Politècnica de Catalunya, Barcelona, Spain

${ }^{21}$ Universidad de Sevilla, Seville, Spain

${ }^{22}$ Istituto Nazionale di Astrofisica - Osservatorio Astronomico di Teramo, Teramo, Italy

${ }^{23}$ Dipartimento di Fisica, Università degli Studi di Bari, Bari, Italy

${ }^{24}$ School of Physics and Astronomy, University of Edinburgh, Edinburgh, United Kingdom

${ }^{25}$ Paul Scherrer Institut (PSI), Villigen, Switzerland

\footnotetext{
*jmoreno53@us.es

†stanislav.valenta@mff.cuni.cz

${ }^{\ddagger}$ gunsing@cea.fr

${ }^{\S}$ Deceased.

${ }^{\mathbb{W}}$ www.cern.ch/ntof.
}

Published by the American Physical Society under the terms of the Creative Commons Attribution 4.0 International license. Further distribution of this work must maintain attribution to the author(s) and the published article's title, journal citation, and DOI. 


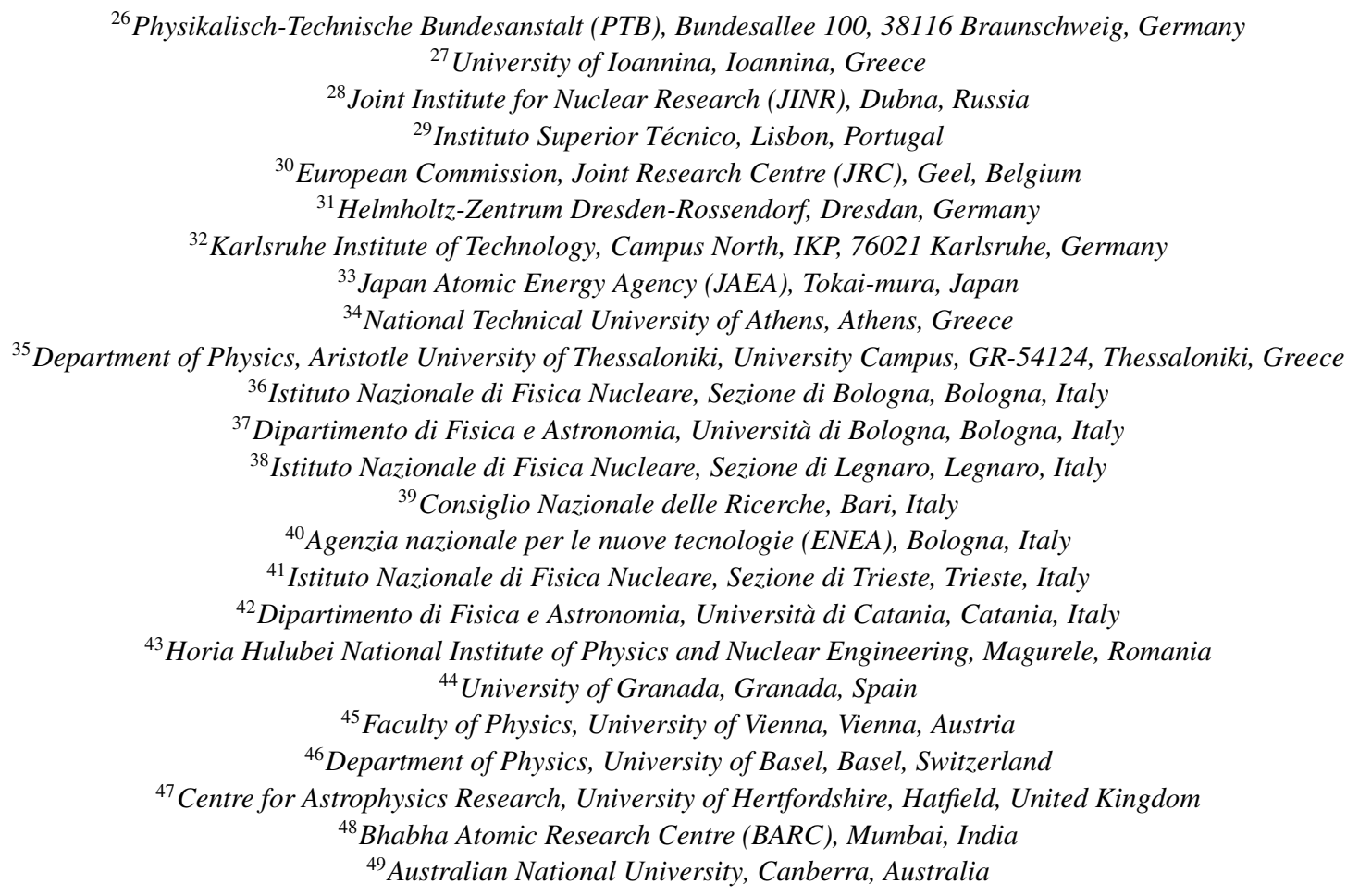

(Received 24 August 2021; accepted 31 January 2022; published 24 February 2022)

Background: The photon strength functions (PSFs) and nuclear level density (NLD) are key ingredients for calculation of the photon interaction with nuclei, in particular the reaction cross sections. These cross sections are important especially in nuclear astrophysics and in the development of advanced nuclear technologies.

Purpose: The role of the scissors mode in the M1 PSF of (well-deformed) actinides was investigated by several experimental techniques. The analyses of different experiments result in significant differences, especially on the strength of the mode. The shape of the low-energy tail of the giant electric dipole resonance is uncertain as well. In particular, some works proposed a presence of the $E 1$ pygmy resonance just above $7 \mathrm{MeV}$. Because of these inconsistencies additional information on PSFs in this region is of great interest.

Methods: The $\gamma$-ray spectra from neutron-capture reactions on the ${ }^{234} \mathrm{U},{ }^{236} \mathrm{U}$, and ${ }^{238} \mathrm{U}$ nuclei have been measured with the total absorption calorimeter of the n_TOF facility at CERN. The background-corrected sum-energy and multi-step-cascade spectra were extracted for several isolated $s$-wave resonances up to about $140 \mathrm{eV}$.

Results: The experimental spectra were compared to statistical model predictions coming from a large selection of models of photon strength functions and nuclear level density. No combination of PSF and NLD models from literature is able to globally describe our spectra. After extensive search we were able to find model combinations with modified generalized Lorentzian (MGLO) E1 PSF, which match the experimental spectra as well as the total radiative widths.

Conclusions: The constant temperature energy dependence is favored for a NLD. The tail of giant electric dipole resonance is well described by the MGLO model of the $E 1$ PSF with no hint of pygmy resonance. The $M 1$ PSF must contain a very strong, relatively wide, and likely double-resonance scissors mode. The mode is responsible for about a half of the total radiative width of neutron resonances and significantly affects the radiative cross section.

DOI: 10.1103/PhysRevC.105.024618

\section{INTRODUCTION}

Nuclear level densities (NLDs) and photon strength functions (PSFs), also called $\gamma$-ray or radiation strength functions, represent average properties of the nucleus in the regime of excitation where individual levels and transition probabilities by $\gamma$ decay are not readily accessible by experimental or theoretical means. They are key ingredients for statistical calculations of the reaction cross sections involving $\gamma$ rays via the Hauser-Feshbach approach [1], like inelastic scattering or neutron capture reactions. These cross sections are important for nuclear network calculations, for example in stellar nucleosynthesis models $[2,3]$ or in nuclear technology applications, 
usually relying on evaluated nuclear data libraries $[4,5]$ or astrophysical databases [6].

Individual levels can be observed and their properties (spin, parity, widths, and eventually decay paths) determined almost exclusively only in two regions: at the lowest excitation energies and in the neutron resonance region just above the neutron separation energy $S_{n}$. The concept of NLD thus has to be used already from an excitation energy well below $1 \mathrm{MeV}$ in odd $\mathrm{U}$ isotopes as the rapidly increasing number of levels prevents their observation. Neutron resonances then serve as an anchor point for the determination of the NLD parameters.

The PSFs for different transition types and multipolarities describe the average transition probability between nuclear levels. Detailed information on PSFs below the neutron separation energy in actinides (needed for cross section calculations) has been a subject of several experimental studies during the last years. They included mainly data from the Oslo technique [7-9] and neutron capture experiments [10,11]. Moreover, there are also data from other experimental techniques including average resonance capture (ARC) [12] or nuclear resonance fluorescence (NRF) $[13,14]$. The two features that strongly influence the $\gamma$ decay and neutron capture cross section are the energy dependence of the giant electric dipole resonance (GEDR) tail with a possible presence of the pygmy resonance and the collective $M 1$ excitation known as the scissors mode (SC) [15-19]. A detailed understanding of these features is important also for the calculation of cross sections for short-lived nuclei. There are many available models of both the PSFs and NLD in the literature [20,21] and their validation as well as obtaining further information on the properties of these quantities is important. At present, literature sources differ on the properties of the scissors mode. In particular its integrated strength in ${ }^{235} \mathrm{U}$ deduced from NRF is about $3 \mu_{N}^{2}$ [13], where $\mu_{N}$ stands for the nuclear magneton, while the analyses of Oslo data [7-9] provide strengths of 8-11 $\mu_{N}^{2}$ for several actinides (consistently for even-even, odd, and odd-odd nuclei). The DANCE data [11] from the Los Alamos Neutron Science Center indicated a lower limit of $11 \mu_{N}^{2}$ for odd uranium isotopes. The calculation therein showed that the presence of a SC with a strength of $\approx 15 \mu_{N}^{2}$ is responsible for about half of the total neutron capture cross section.

In the present paper we explore data from the $4 \pi$ total absorption calorimeter (TAC) of the n_TOF facility at CERN for three uranium isotopes. Data for these nuclei have been taken previously and analyzed in terms of neutron capture cross sections for ${ }^{234} U[22,23],{ }^{236} U[24,25]$, and ${ }^{238} U[26,27]$. Most of the used experimental procedures are detailed in those references and we only summarize the main aspects here. We reanalyzed these data and extracted sum-energy and multistep cascade (MSC) spectra. These spectra were then confronted with predictions of statistical model simulations of $\gamma$-ray cascades based on several models for NLDs and PSFs. Such a comparison provides significant constraints on the NLD and PSF models. We found that models available in the literature needed to be improved. Based on extensive simulations we were able to find PSFs common for all three nuclei. A first intermediate report showed preliminary results on the nucleus ${ }^{234} \mathrm{U}$ [28] while work on all three nuclei is reported

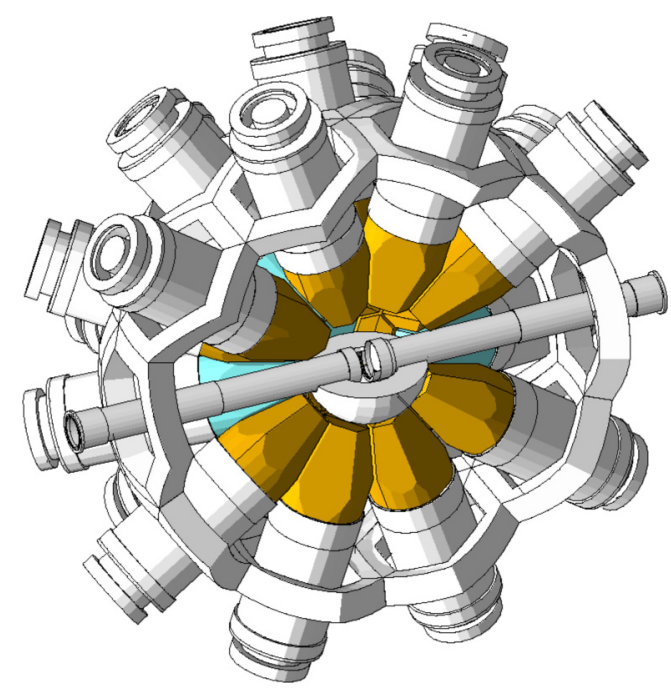

FIG. 1. One hemisphere of the TAC showing the $\mathrm{BaF}_{2}$ crystals and photomultipliers mounted in the support structure. The vacuum time-of-flight tube traversing the detector is split at the center of the TAC where the sample holder in air is positioned, surrounded by the neutron absorber of which only the lower half is shown.

in Ref. [29]. Since then additional models have been used as well and the final results are presented here. An important conclusion of our analysis is a verification of results obtained from the analysis of MSC spectra from the DANCE detector [11], the $4 \pi$ calorimeter with $160 \mathrm{BaF}_{2}$ crystals operated at the Los Alamos Neutron Science Center. As the MSC spectra from the independent $n_{-}$TOF experiment are used for the present extensive PSF analysis, such a verification forms an important confirmation of the employed method.

The paper is organized as follows: the experimental setup and data reduction are described in Sec. II while simulations within the statistical model in Sec. III. Sections IV and V present results coming from the comparison of experimental spectra with their simulated counterparts and the comparison of integral quantities to available literature data, respectively. The conclusions are then given in Sec. VI.

\section{EXPERIMENT AND DATA REDUCTION}

\section{A. Experimental setup}

The n_TOF neutron-time-of-flight facility at CERN $[30,31]$ uses a white neutron spectrum produced by a pulsed proton beam of $20 \mathrm{GeV} / c$ supplied by the Proton Synchrotron impinging on a lead spallation target. Neutron capture experiments on ${ }^{234} \mathrm{U},{ }^{236} \mathrm{U}$, and ${ }^{238} \mathrm{U}$ were performed with the total absorption calorimeter (TAC) [27,32] consisting of 40 $\mathrm{BaF}_{2}$ crystals, lined with carbon shells doped with ${ }^{10} \mathrm{~B}$, in a $4 \pi$ configuration surrounding the capture sample placed in the neutron beam. The high-efficiency calorimeter is placed at a distance of about $185 \mathrm{~m}$ from the spallation target in the time-of-flight station EAR1 [31].

In Fig. 1 the detector setup shows one hemisphere of the TAC with the vacuum neutron flight tube traversing it. A sample holder is placed in the center of the TAC where the vacuum tube is interrupted by mylar windows. The lower 
TABLE I. Summary of the characteristics of the samples and experimental conditions for each measurement. Additional details are given in the text.

\begin{tabular}{lccc}
\hline \hline & ${ }^{234} \mathrm{U}$ & ${ }^{236} \mathrm{U}$ & ${ }^{238} \mathrm{U}$ \\
\hline Mass $(\mathrm{mg})$ & 32.7 & 338 & 6125 \\
Areal density $\left(10^{-4}\right.$ atoms $\left./ \mathrm{b}\right)$ & 1.07 & 10.9 & 9.56 \\
Canning & $\mathrm{Ti}$ & $\mathrm{Al}$ & $\mathrm{Al}$ \\
$\mathrm{S}_{n} \approx Q(\mathrm{MeV})$ & 5.297 & 5.126 & 4.806 \\
Resolution $(\%)(0.9 \mathrm{MeV})$ & 14.5 & 16.5 & 16.5 \\
\hline \hline
\end{tabular}

half of the surrounding neutron absorber, intended to reduce the main source of background produced by sample-scattered neutrons, is shown as well. The absorber for the ${ }^{234} \mathrm{U}$ experiment consisted of an inert nonflammable ${ }^{6} \mathrm{Li}$-enriched salt produced from carbonic and dodecanedioic acids encapsulated by $0.5 \mathrm{~mm}$ of $\mathrm{Al}$, while the absorber used for the ${ }^{236} \mathrm{U}$ and ${ }^{238} \mathrm{U}$ experiments was produced as a self-supporting borated polyethylene containing $5 \%$ of ${ }^{10} \mathrm{~B}$. Energy calibration and resolution determination of individual $\mathrm{BaF}_{2}$ crystals were performed using spectra from ${ }^{137} \mathrm{Cs}$ and ${ }^{88} \mathrm{Y}$ radioactive sources. The crystal resolution was on average $14.5 \%$ for $0.9 \mathrm{MeV} \gamma$ rays at the time of the ${ }^{234} \mathrm{U}(n, \gamma)$ measurement and slightly worsened to $16.5 \%$ during the ${ }^{236} \mathrm{U}(n, \gamma)$ and ${ }^{238} \mathrm{U}(n, \gamma)$ experiments.

All three uranium samples were highly enriched (>99\%). No further information on the isotopic composition of the 32.7 mg ${ }^{234} \mathrm{U}$ sample was available, the measured spectra did not show any isotopic impurity. For the $338 \mathrm{mg}{ }^{236} \mathrm{U}$ sample the enrichment was $99.85 \%$ and contained small amounts of ${ }^{235} \mathrm{U}$ $(0.05 \%)$ and ${ }^{238} \mathrm{U}(0.1 \%)$. Both samples were provided by the Institute of Physics and Power Engineering in Obninsk as $10 \mathrm{~mm}$ diameter disk-shaped encapsulated pressed pellets of uranium oxide. The $6.125 \mathrm{~g}{ }^{238} \mathrm{U}$ sample supplied by JRCGeel was a highly enriched $\left(<1 \mathrm{ppm}^{234} \mathrm{U},<11 \mathrm{ppm}^{235} \mathrm{U}\right.$, and $\left.<1 \mathrm{ppm}^{236} \mathrm{U}\right)$ metallic foil, encapsulated in $60 \mu \mathrm{m}$ of aluminum and about $75 \mu \mathrm{m}$ of Kapton, with a nearly rectangular shape of about $53.9 \times 30.3 \mathrm{~mm}^{2}$, therefore fully covering the neutron beam diameter of approximately $20 \mathrm{~mm}$. The effective area of $1621.22 \mathrm{~mm}^{2}$ was determined with a microscope-based measurement system. Given the cross sections and the selected neutron energy ranges we have used in this work, we assumed that the impurities have a negligible impact on the extracted spectra. A summary of the most relevant sample and detector parameters is given in Table I.

\section{B. Data processing}

The signals from each individual $\mathrm{BaF}_{2}$ crystal were sampled using digitizers at a rate of $5 \mathrm{e} 8$ samples/s. The signal consists of two components with decay times of $0.7 \mathrm{~ns}$ (fast) and $630 \mathrm{~ns}$ (slow). The fast and slow components allow the precise determination of the arrival time and the deposited energy, respectively. The ratio of their amplitudes then allows the discrimination of $\gamma$ rays from background $\alpha$ particles caused by the natural radioactivity of the $\mathrm{Ra}$ contaminant in the $\mathrm{BaF}_{2}$ crystals $[22,33]$. In the analysis we considered only signals corresponding to a deposited energy above a $250 \mathrm{keV}$ threshold, which is slightly above the hardware one.

The $\gamma$-ray signals within a coincidence window of $20 \mathrm{~ns}$ were considered to belong to the same TAC event. Each TAC event is characterized by (i) a corresponding neutron energy determined by the time-of-flight technique from the time of signal arrival, (ii) a number of firing crystals called crystal multiplicity $m$, and (iii) energies deposited in individual crystals, $E_{i}$. The time-of-flight spectra for the $(n, \gamma)$ reactions on ${ }^{234} \mathrm{U},{ }^{236} \mathrm{U}$, and ${ }^{238} \mathrm{U}$ are shown in Fig. 2.

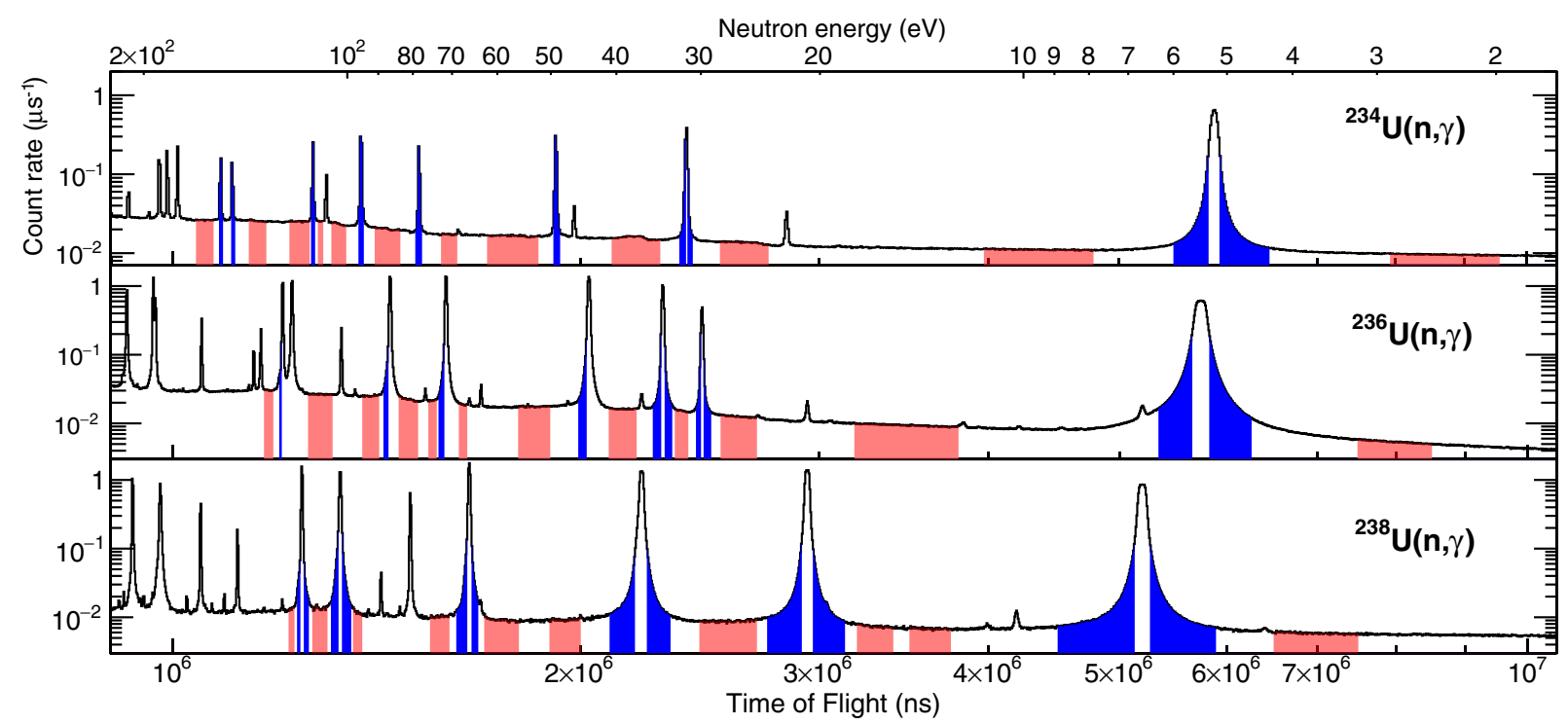

FIG. 2. The time-of-flight spectra for the $(n, \gamma)$ reactions on ${ }^{234} \mathrm{U},{ }^{236} \mathrm{U}$, and ${ }^{238} \mathrm{U}$ for $m \geqslant 2$ TAC events. The top axis shows the corresponding neutron-energy scale. The blue regions are used for the analysis while the red ones correspond to the windows for the background subtraction; see text for details. 


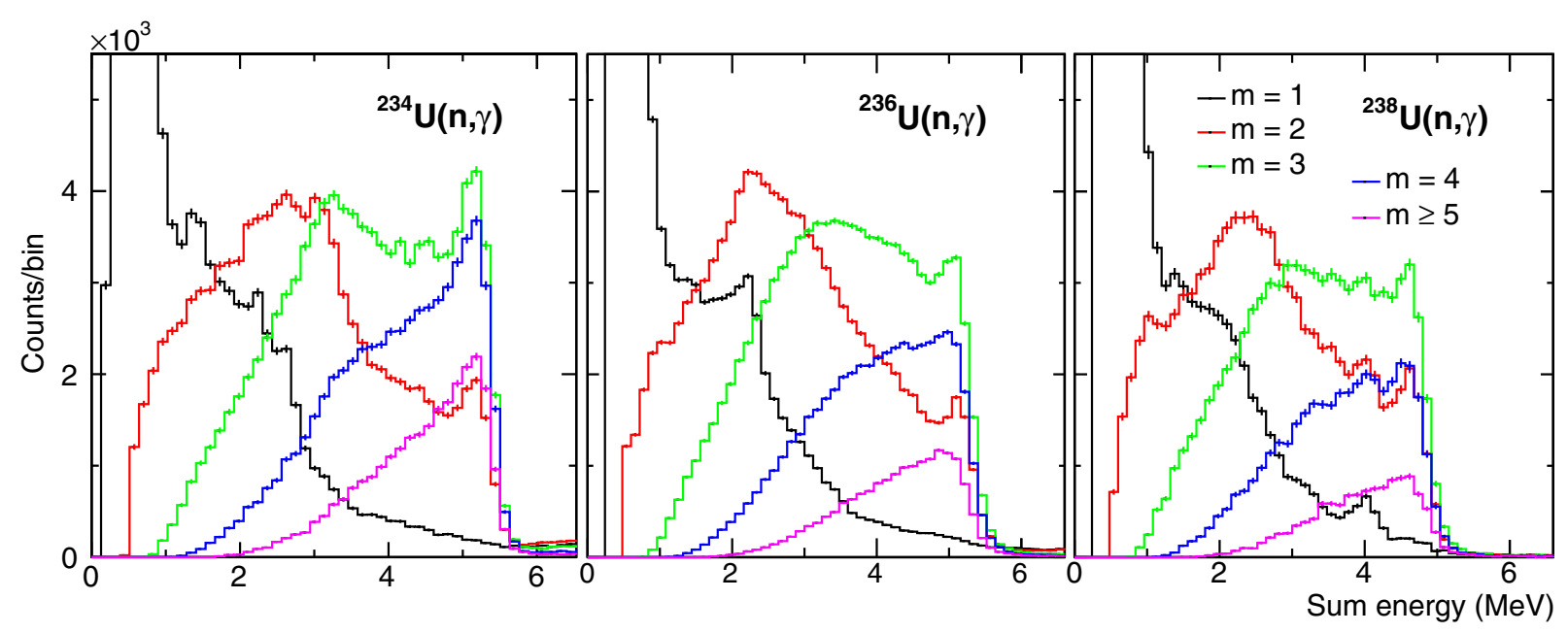

FIG. 3. Sum energy spectra for the first resonance in $(n, \gamma)$ reaction on ${ }^{234} \mathrm{U},{ }^{236} \mathrm{U}$, and ${ }^{238} \mathrm{U}$, not corrected for background, for different crystal multiplicity criteria. The counts for $m=1$, attributed mainly to background events, go up as high as $120 \times 10^{3}$ counts/bin. The $Q$ values of the ${ }^{234,236,238} \mathrm{U}(n, \gamma)$ reactions are 5.3, 5.1, and 4.8 MeV respectively, in practice equivalent to the $S_{n}$ values listed in Table I.

Only cascades originating from well-resolved $s$-wave $\left(J^{\pi}=\frac{1}{2}^{+}\right)$resonances with sufficient statistics were further used. To minimize the event pileup due to high local count rate and a strong dead time we excluded the peak regions of the resonances and used only regions indicated in Fig. 2. The eight resonances at 5.16, 31.1, 48.6, 77.4, 94.3, 111.1, 146.3, and $152.2 \mathrm{eV}$, seven at 5.45, 29.8, 34.1, 43.9, 71.5, 86.5, and $124.9 \mathrm{eV}$, and six at $6.67,20.9,36.7,66.0,102.6$, and $116.9 \mathrm{eV}$ from ${ }^{234,236,238} \mathrm{U}(n, \gamma)$, respectively, were analyzed. In reality, the selected neutron-energy regions are still slightly influenced by the dead-time effects. The correction for dead-time and pileup effects for the TAC are far from trivial [34]. However, as this impact is very similar for all the regions we have decided not to correct the experimental spectra but instead consider this effect in the simulations. For this purpose we modeled the average observed distribution of consecutive detected events, which in the ideal case contains a step function representing the dead time, with a parameterizable sigmoid function and an exponential $[22,29]$.

For a given neutron resonance two kinds of spectra were constructed from individual cascades for a given multiplicity $m$ criterion: the energy spectrum summed over all crystals $E_{s}=\sum_{i} E_{i}$, hereafter called sum-energy spectrum, and the multistep cascade (MSC) spectrum, which corresponds to the energies $E_{i}$ deposited in each of the $m$ individual crystals within a TAC event. The sum-energy spectra before background subtraction are shown in Fig. 3 for the first resonance of each nucleus. In the case of complete cascade detection the $E_{s}$ would be equal to the $Q$ value of the reaction, $Q=$ $S_{n}+E_{n} \approx S_{n}$, with $E_{n}$ being negligible relative to $S_{n}$. The Compton scattering and the finite efficiency of the detectors makes a significant fraction of the cascades contribute to lower energies. At least for several multiplicities a peak near the $Q$ value (with a width of a few hundreds $\mathrm{keV}$ given by detector energy resolution) with a sharp fall above is visible.

The MSC spectra were constructed only from TAC events with deposited energy sum near $S_{n}$, specifically for
$E_{s}=5.0-5.6 \mathrm{MeV}$, 4.9-5.3 MeV, and 4.5-4.8 MeV for the ${ }^{234,236,238} \mathrm{U}$ samples, respectively. Those windows encompass the resolution-broadened $Q$ values and were also adjusted in order to have sufficient statistics in the MSC spectra.

A bin width of $130 \mathrm{keV}$, which is close to the energy resolution of crystals for low $E_{\gamma}$, was used. For a background subtraction of spectra from individual resonances we used linear interpolation from neighboring off-resonance regions shown in Fig. 2; for details on background subtraction see Ref. [29]. All spectra for a given resonance were normalized by one common factor given by the integral of the $m \geqslant 2$ sum-energy spectrum in the corresponding aforementioned $E_{s}$ range.

The background corrected spectra from ${ }^{234} \mathrm{U}(n, \gamma)$ resonances are shown in Fig. 4. Analogous figures for ${ }^{236,238} \mathrm{U}(n, \gamma)$ can be found in the Supplemental Material [35]. The spectra may not necessarily be consistent within their counting uncertainties since there is an additional spread due to the Porter-Thomas fluctuations [36] of individual transition intensities. This additional spread is clearly observed and confirmed by a maximum likelihood fit assuming a normal distribution of the MSC intensity. The effect is mainly visible in the $m=2$ MSC spectra, for example in ${ }^{234} \mathrm{U}(n, \gamma)$ in the peaks around 0.7 and $4.5 \mathrm{MeV}$ as shown in Fig. 4. In other energy domains it is to most extent masked by the uncertainties of individual spectra.

For a comparison of experiment with predictions we decided not to use spectra from individual resonances. The restricted number of resonances does not allow one to reliably determine the properties of the distribution from a maximum likelihood fit as in Ref. [37]. We thus calculated the unweighted average and standard deviation of the set; see Fig. 4. The $m=1$ spectra are dominated by the background (see Fig. 3) and are not considered in the analysis. Spectra for $m>4$ do not show any interesting structures (see Fig. 4), and the intensity is rapidly decreasing with $m$; there are practically no TAC events with $m \geqslant 7$. 


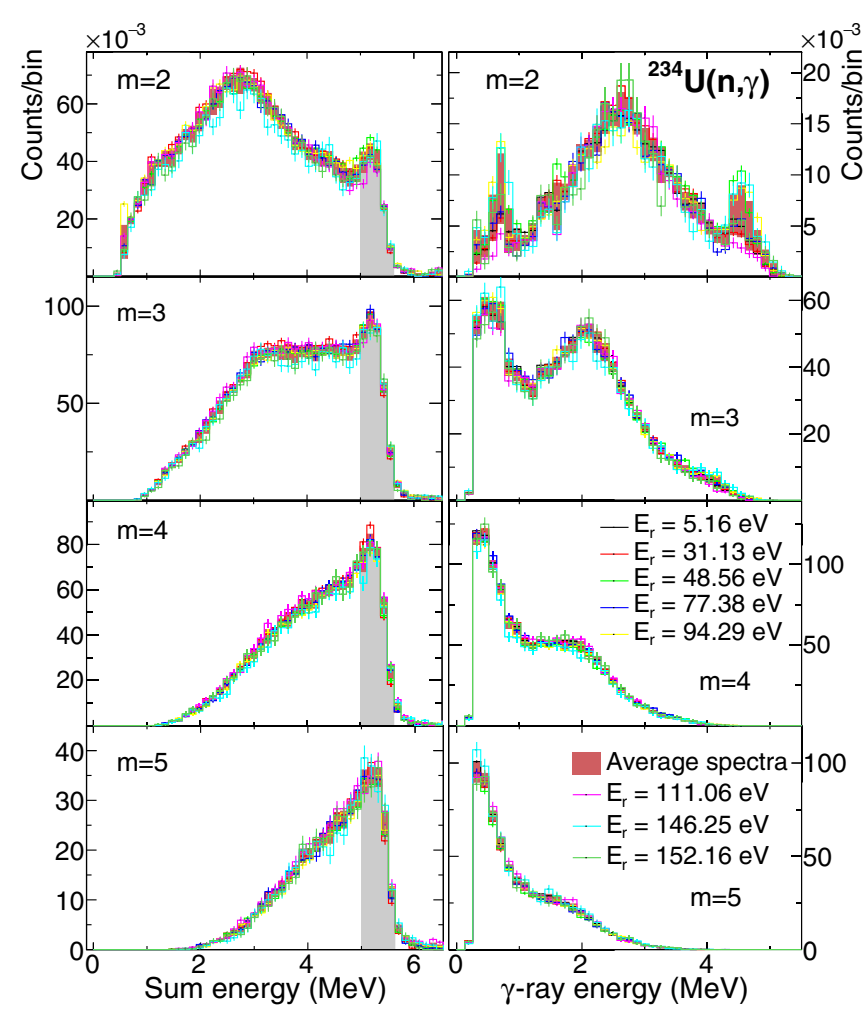

FIG. 4. The sum-energy and MSC spectra for individual resonances and multiplicities $m=2-5$ after background subtraction for ${ }^{234} \mathrm{U}(n, \gamma)$. The label " $\gamma$-ray energy" refers to the deposited energy in any crystal. The "Average spectra" band represents the average of resonances plus/minus one standard deviation representing the fluctuation among the resonances. The shaded interval depicts the $E_{s}$ range used for the construction of MSC spectra.

From the available information on TAC events we can construct also other observables, e.g., the commonly used multiplicity distribution of detected TAC events; see experimental results in Fig. 5. However, as this quantity is inherently contained in both the sum-energy and MSC spectra and was found to be less sensitive to the changes in NLD and PSF models, we do not concentrate on its comparison with simulated
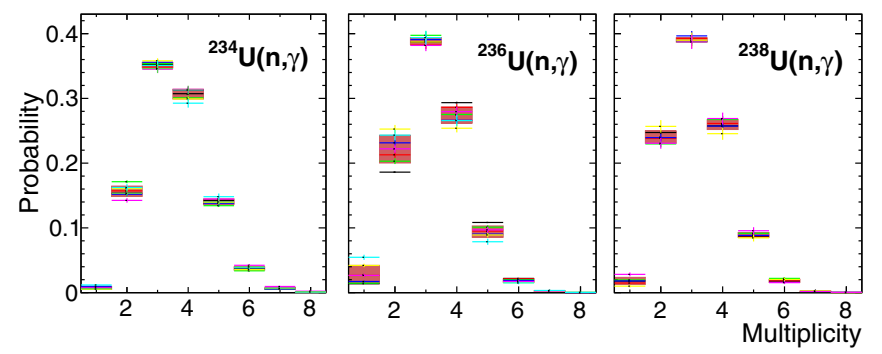

FIG. 5. Crystal multiplicity distribution for each uranium isotope from the same $E_{s}$ energies as used for the construction of MSC spectra. The individual resonances after background subtraction are plotted following the color scheme in the legend of Fig. 4. The average spectra and the standard deviation are represented by the band. multiplicity distributions. Nevertheless, a few figures can be found in the Supplemental Material [35].

\section{SIMULATIONS}

The sum-energy and MSC spectra arise from a complex interplay between the PSFs, NLD, and detector response. This fact prevents a direct extraction of the PSFs and/or NLD from our data. However, we can learn about these quantities by comparing the experimental spectra with their simulated counterparts based on various PSF and NLD models. This trial-and-error approach is the only reasonable way to test the available models. Attempts to fine tune the models then usually require extensive simulations. The resulting model combinations will in that case provide the best match with the experimental data but do not necessarily form a unique or optimum solution.

\section{A. Algorithms}

Individual $\gamma$-ray cascades were simulated within the statistical approach utilizing the Monte-Carlo DICEBOX code [38]. The fluctuations between artificial nuclei as generated by DICEBOX are depicted by the color $\pm 1 \sigma$ bands when comparing the simulated spectra to their experimental counterparts. We simulated 20 artificial nuclei with $10^{5}$ cascades within each. Many different combinations of models of NLD and $E 1, M 1$, and $E 2$ PSFs were used. As the description of $\gamma$ decay within the statistical approach is surely inadequate at the lowest excitation energies, the information on discrete levels and their decay was taken from the ENSDF database [39-41] up to excitation energies of 820,760 , and $830 \mathrm{keV}$ for the compound nuclei ${ }^{235} \mathrm{U},{ }^{237} \mathrm{U}$, and ${ }^{239} \mathrm{U}$ respectively.

The TAC response to these cascades was obtained using the code based on the GEANT4 package [42]. We implemented the full geometry of the supporting structures, beam pipes, detectors, neutron absorber, and uranium samples in detail [43]. We implemented also the energy resolution of $\mathrm{BaF}_{2}$ crystals and the dead time process following the experimentally determined distribution of consecutive pulses [22,29]. Simulated spectra thus should be directly comparable to the measured ones. The normalization of simulated spectra was done in the same way as for the experimental ones.

A precise statistical quantification of the agreement between the simulated and experimental spectra cannot be made without time-consuming simulations due to a priori unknown highly nontrivial correlations between individual bins of the MSC spectra. We tested a simple numerical scoring function of the spectral similarity or goodness of fit, but it was found to be inconclusive mainly because of the large associated uncertainties, except for obvious discordant cases. In this paper we mostly kept the PSF and NLD model combinations which were not in undebatable disagreement with the measured spectra.

It is to be stressed that the predicted spectra are not sensitive to the absolute values of PSFs if the $E_{\gamma}$-dependent ratios of PSFs for different transition types are kept the same. So, we can rather probe the $E_{\gamma}$ dependence of the PSFs and their relative contributions than the absolute PSF values. The only 
TABLE II. Parameters of PSF models used in simulations. The $E 1$ PSF parameters correspond to the double-peaked GEDR energy $E$, width $\Gamma$, and maximum cross section $\sigma$. The same quantities then characterize the double resonance SC and the SF resonances in the $M 1$ PSF. $\operatorname{MGLO}(k)$ is used with a $T=0.3 \mathrm{MeV}$.

\begin{tabular}{|c|c|c|c|c|c|c|c|c|c|c|c|c|c|c|c|}
\hline $\begin{array}{l}\text { Model } \\
\text { combination }\end{array}$ & \multicolumn{6}{|c|}{$E 1 \mathrm{PSF}$} & \multicolumn{9}{|c|}{$M 1 \mathrm{PSF}$} \\
\hline RIPL-3 [21] & 11.11 & 1.12 & 243.3 & 13.41 & 4.98 & 426 & & & & & & & 6.61 & 4.00 & 2.35 \\
\hline Oslo $^{\mathrm{a}},{ }^{\mathrm{b}}$ [7] & 11.40 & 4.20 & 572 & 14.40 & 4.20 & 1040 & 2.15 & 0.80 & 0.45 & 2.90 & 0.60 & 0.40 & 6.61 & 4.00 & 7.00 \\
\hline Oslo $^{a},{ }^{c}$ [7] & 11.40 & 4.20 & 572 & 14.40 & 4.20 & 1040 & 2.00 & 0.80 & 0.40 & 2.80 & 1.20 & 0.30 & 6.61 & 4.00 & 7.00 \\
\hline
\end{tabular}

${ }^{\mathrm{a}} E 1 \mathrm{PSF}$ contained an additional Lorentzian at $7.3 \mathrm{MeV}$ with width of $2 \mathrm{MeV}$ and maximum cross section of $15 \mathrm{mb}$.

${ }^{\mathrm{b} 235,237} \mathrm{U}$.

${ }^{\mathrm{c} 239} \mathrm{U}$.

${ }^{\mathrm{d}}$ The value was taken from systematics in the RIPL-3 database [21] and was not adjusted.

quantity from simulations that depends on the absolute PSF values is the total radiative width $\Gamma_{\gamma}$.

\section{B. Tested models}

There are many models of PSFs and NLD available in the literature; see, e.g., reviews $[20,21,44]$. We decided to check our spectra against predictions based on a few of them which are either proposed in the recent reviews or describe relevant experimental data, namely,

(i) one of the widely used model combinations available in the RIPL-3 database [21] consisting of the analytical generalized Lorentzian (GLO) model for $E 1$ PSF in combination with the spin-flip (SF) Lorentzian for $M 1$ PSF coupled with the constant-temperature (CT) NLD model [45], hereafter called RIPL-3,

(ii) the PSF models proposed in the recent review [20] based on microscopic calculations combined with some phenomenological parts used in conjunction with microscopically Hartree-Fock-Bogoliubov (HFB) based plus combinatorial NLD [46], hereafter called IAEA-19,

(iii) the original model interpretation of the Oslo PSF [7] and NLD [47], based on the $(d, t){ }^{237} \mathrm{U}$ and $(d, p){ }^{239} \mathrm{U}$ data, hereafter called $O$ slo, consisting of the enhanced generalized Lorentzian (EGLO) E1 model, the Lorentzian SC and SF $M 1$ modes, and the CT NLD model from Ref. [47],

(iv) the model combination, hereafter called DANCE, that reasonably described the MSC spectra in the U isotopes from DANCE experiment [11] consisting of the modified generalized Lorentzian (MGLO) E1 PSF model, the Lorentzian SC and SF $M 1$ modes, and the CT NLD [45].

The parameters of the PSF resonance structures can be found in Table II. The parameters of the phenomenological parts of the IAEA-19 model combination are $f_{0}=1 \times 10^{-10}$ $\mathrm{MeV}^{-4}, E_{0}=4 \mathrm{MeV}$ for $E 1 \mathrm{PSF}$ and $C=1 \times 10^{-8} \mathrm{MeV}^{-3}$ and $\eta=0.8 \mathrm{MeV}^{-1}$ for $M 1 \mathrm{PSF}$ and correspond to one of the options proposed in $[20,48]$.
The PSF and NLD models for ${ }^{239} \mathrm{U}$ are shown in Figs. 6 and 7, respectively. The PSFs for two of these model combinations-Oslo and DANCE-were chosen such that they follow the exact form of the Brink hypothesis [49] saying that the PSFs are only a function of $E_{\gamma}$. This is achieved by describing the $M 1$ modes by Lorentzians and by using a constant value of temperature $T$ in the $E 1$ models. The GLO model of the RIPL-3 combination contains a dependence on nuclear temperature $T$, which is considered a function of the excitation energy in accord with Ref. [21]. The E1 PSF of IAEA-19 shows a dependence, albeit very weak, on the excitation energy through the phenomenological part.

As is evident from the comparison in Sec. IV, the description of our experimental spectra is not perfect with any of these models. We thus decided to make an extensive search to find a model combination which would ideally provide a perfect agreement with the experimental data. Based on the promising results of the MSC spectra analysis from the DANCE experiment [11] and on the adjustability of the MGLO model [52] we decided to dominantly exploit this $E 1$ PSF model in combination with the SF and SC $M 1$ modes (each $M 1$ resonance term was described by a Lorentzian in the PSF). This combination of PSFs was tested in conjunction with different NLD models: the CT NLD model using two different parametrizations [45,50], the Back-shifted Fermi gas (BSFG) NLD model [50], as well as the microscopic NLD [46]; see Fig. 7. The other $E 1$ model possibilities were tested as well, albeit not in such detail; see the Supplemental Material [35].

There are free parameters in both the $E 1$ and $M 1$ PSF models. In the latter case we considered the energies, widths, and maximum cross sections of the SC resonance terms, as well as the maximum cross section of the SF resonance, as the free parameters. We almost exclusively used a doubleresonance SC; simulations with a single-resonance SC did not lead to an agreement comparable to that reached with the double-resonance one for all three isotopes. The MGLO model depends on a few parameters besides the GEDR energies $E$, widths $\Gamma$, and maximum cross sections $\sigma$. The parameter $k$, see the Supplemental Material [35], was considered a free one in our analysis. As mentioned above, the 


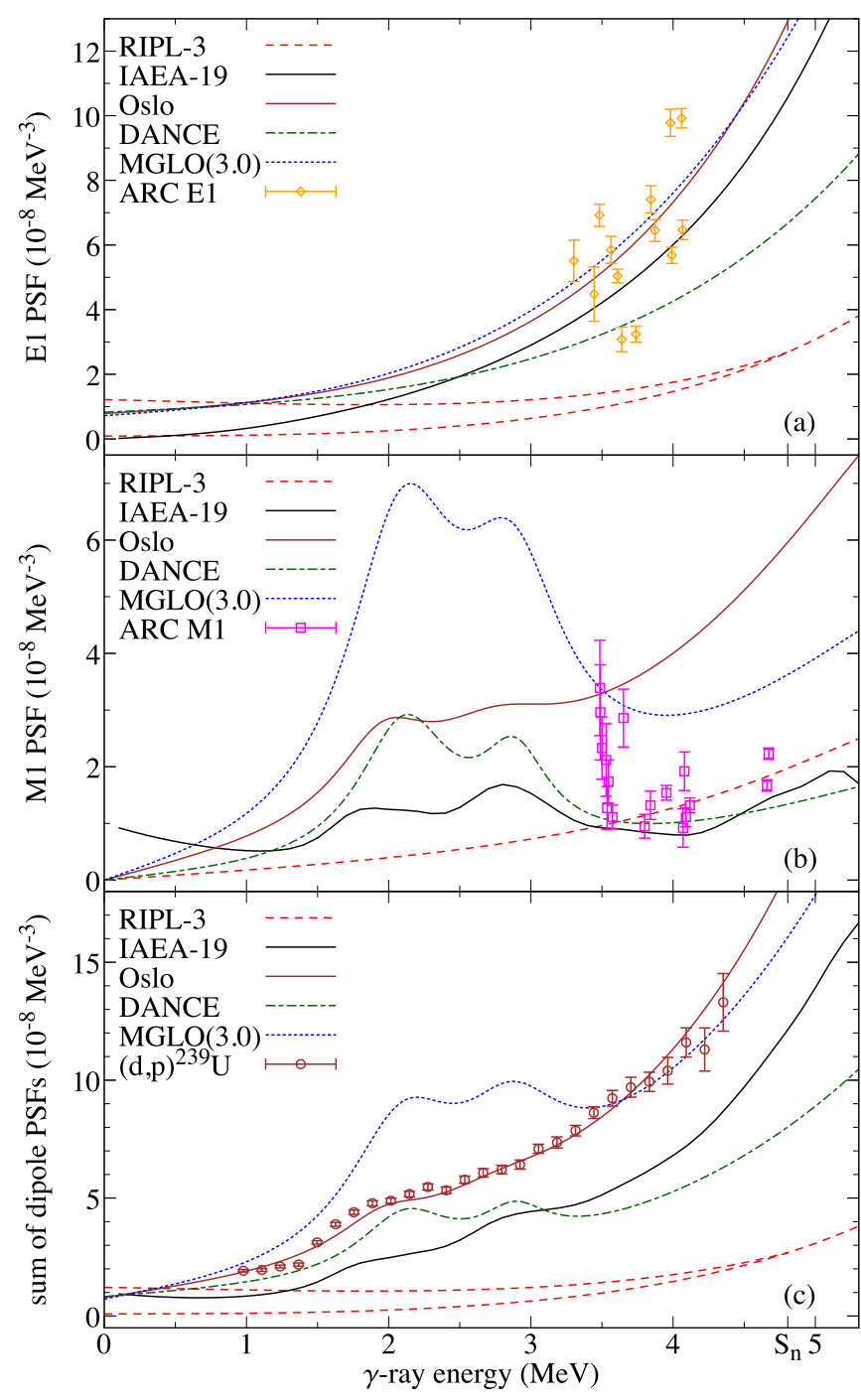

FIG. 6. Photon strength functions of ${ }^{239} U$ as a function of $\gamma$-ray energy for some of the models used in our simulations. The panels (a) and (b) display the $E 1$ and $M 1$ PSFs, respectively. For the model parameters see Table II. The IAEA-19 $E 1$ and $M 1$ PSFs were calculated by Goriely et al. [20] and the average resonance capture data were compiled by Kopecky [12]. For the temperature dependent GLO model the lower and upper curve correspond to excitation above ground state and decay from the capturing state, respectively. The panel (c) shows the sum of the $E 1$ and the corresponding $M 1$ PSF [from panels (a) and (b)] compared to the Oslo data from the $(d, p){ }^{239} \mathrm{U}$ reaction [7].

MGLO model further contains a dependence on nuclear temperature $T$, which can be either considered a free parameter or dependent on the excitation energy in accord with the original formulation [52]. Both options were considered in our analysis with a few values of constant $T$ tested. The GEDR parameters could be taken from the literature. However, with such a parametrization the MGLO model does not usually reproduce the photoabsorption data. We thus decided to fit the photoabsorption data from Ref. [53] in the range 9-16 MeV by the MGLO model for each set of $k$ and $T$ parameters. The resulting GEDR parameters are listed in Table II and the

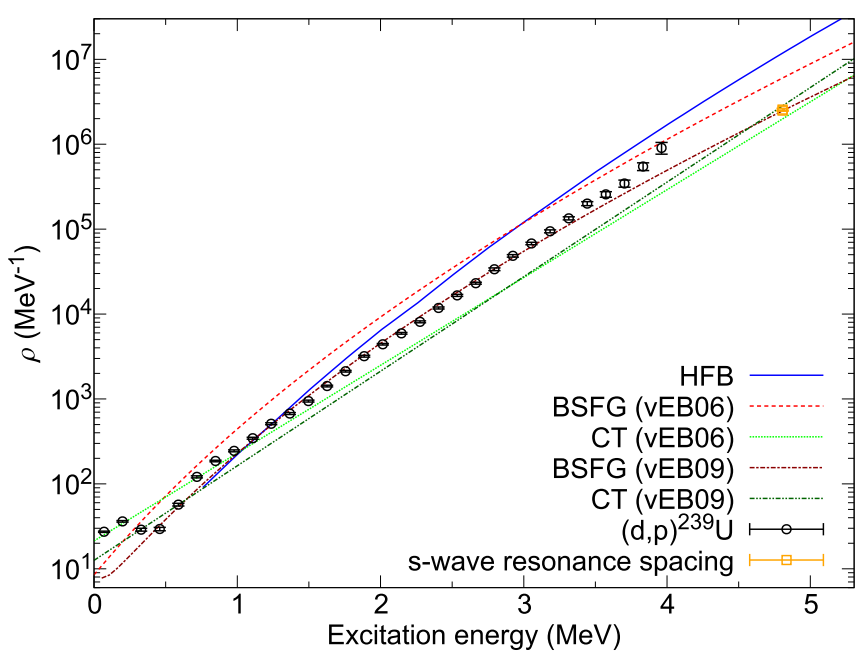

FIG. 7. Spin- and parity-summed level density for ${ }^{239} \mathrm{U}$ according to the CT and BSFG models and the HFB calculations [46]. The parameters of the CT and BSFG NLDs were taken from Refs. [45,50], denoted as (vEB06) and (vEB09) respectively. The experimental data from the Oslo method [7] are shown. Note that the differences at $S_{n}$ stem from different spin distributions in the models. The point corresponding to the $s$-wave resonance spacing [51] was converted using the spin distribution with the spin-cutoff parameter from Ref. [50]. The significant deviation of the Oslo data at higher energies hints to the normalization issue, which was discussed in detail by Ullmann et al. [11].

Supplemental Material [35]. These parameters slightly differ from the available ones coming from the fits of photoabsorption data with different $E 1$ PSF models.

The E2 transitions are believed to play a marginal role in the statistical decay of the nucleus. In all model combinations we used an E2 PSF representing the giant electric quadrupole resonance by a single Lorentzian with parameters from systematics in Ref. [54].

\section{COMPARISON OF SPECTRA}

The common features of the experimental spectra strongly indicate, under an assumption of smooth behavior of the NLD, a presence of a resonant structure in the PSF peaked between 2 and $3 \mathrm{MeV}$. These common features are namely (i) the shape of the continuum of $m=2$ sum-energy spectra with the maxima between 2 and $3 \mathrm{MeV}$, (ii) the change of the slope of $m=3$ sum-energy spectra at $\approx 3 \mathrm{MeV}$, (iii) the shape of $m=2$ MSC spectra showing an accumulation of intensity around their midpoints, and (iv) the bump at $\approx 2 \mathrm{MeV}$ in $m=3$ MSC spectra; see Fig. 4. Note also that the features (i) and (iii) change with different $S_{n}$ while features (ii) and (iv) are relatively stable.

Within our trial-and-error approach we tested the model combinations from the literature (Sec. IV A), tried to fine-tune the PSFs to match the experimental spectra (Sec. IV B) and performed a number of tests to investigate the sensitivity of our data, (Sec. IV C). As mentioned above, we decided not to compare $m=1$ spectra. Although we checked the simulated spectra against their experimental counterparts for $m=2-6$, 


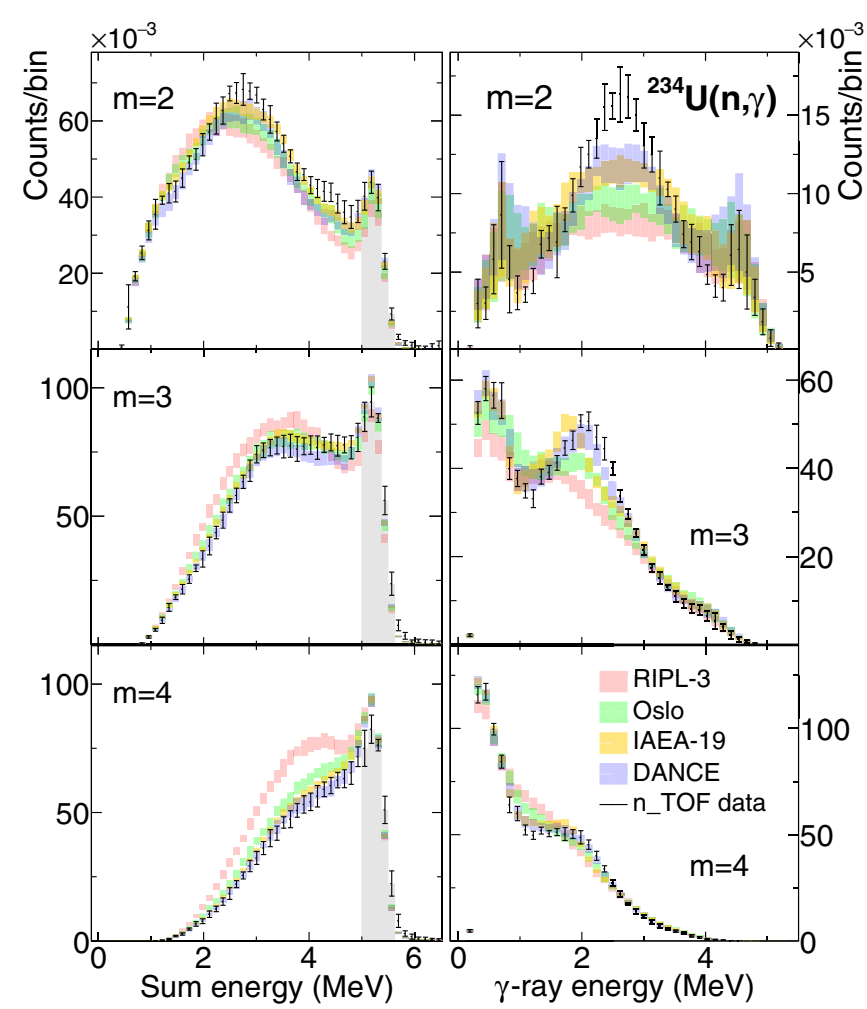

FIG. 8. Comparison of ${ }^{234} \mathrm{U}(n, \gamma)$ sum-energy (left) and MSC (right) spectra using the model combinations RIPL-3, IAEA-19, Oslo, and DANCE. The label " $\gamma$-ray energy" refers to the deposited energy in any crystal.

for the sake of clarity we show only comparison for $m=2-4$. The comparison between the experimental and simulated multiplicity distributions for several model combinations can be found in the Supplemental Material [35].

\section{A. Model combinations from literature}

The average experimental sum-energy and MSC spectra are for ${ }^{234} \mathrm{U}(n, \gamma)$ compared to their simulated counterparts for the model combinations RIPL-3, IAEA-19, Oslo, and DANCE in Fig. 8. Analogous figures for ${ }^{236,238} \mathrm{U}(n, \gamma)$ can be found in the Supplemental Material [35]. The figures indicate that predictions from none of the model combinations from literature match our spectra globally, although the DANCE and IAEA-19 combinations present the best results. In reality, the results depend on the specific nucleus and it might (accidentally) happen that a good agreement is achieved for some spectra. More pronounced differences are usually found in the MSC rather than the sum-energy spectra.

A significant disagreement for the RIPL-3 model combination, namely an absence of structures in MSC spectra [aforementioned features (iii) and (iv)] is evidently a consequence of the complete absence of the SC. This is not really surprising as previous experiments, including analogous DANCE experiment [11], already prove that the SC is necessary for a reasonable description of $\gamma$ decay of highly excited uranium compounds.
Although the model combinations IAEA-19, Oslo, and DANCE contain the SC, their overall disagreement is mostly (but not only) caused by a too low contribution of the SC. In practice, the predicted MSC spectra are very sensitive to the SC position and strength distribution. We can conclude that the available model combinations do not provide a consistent satisfactory description of all our experimental spectra. We thus decided to search for a model combination better reproducing our spectra. Results of this search are presented below.

\section{B. Search for optimal PSFs}

Altogether, hundreds of simulations with different MGLO and scissors mode parametrizations in combination with the above-mentioned NLD models have been compared to the average experimental spectra. The search led to common $E 1$ and $M 1$ PSFs for all three nuclei, which globally describe all the spectra much better than any of the literature model combinations. The achieved level of agreement between simulations and experiment is presented in Fig. 9 for one particular parameter set of the MGLO model with $k=1.8$ and fixed $T=0.3 \mathrm{MeV}$ in combination with the CT NLD from [50]. The remaining parameters of the PSFs are listed in Table II.

As mentioned in Sec. III A simulated spectra are completely independent of absolute PSF scales providing that the $E_{\gamma}$-dependent ratios of the PSFs for the involved transition types are fixed. An agreement comparable to that in Fig. 9 can thus be achieved by an infinite number of PSF sets. One possibility is to multiply dipole PSFs by the same constant factor, ${ }^{1}$ but such a scaling affects the ability to describe the photoabsorption data as well as the total radiative width $\Gamma_{\gamma}$, which is discussed below. Another possibility is to change the parameter $k$ of the MGLO E1 model with a simultaneous adjustment of the SC parameters. The photoabsorption data can be well fitted by the MGLO model with $T=0.3 \mathrm{MeV}$ for a range of $k \approx 1.0-4.0 .^{2}$ The influence of these changes on $\Gamma_{\gamma}$ values is presented below. Additional comparisons of simulated to experimental spectra with different values of $k$, together with the PSF parameters, are available in the Supplemental Material [35].

There is a priori no reason for using $T=0.3 \mathrm{MeV}$. However, data from the Oslo method usually yield a value of $T \approx$ $0.2-0.4 \mathrm{MeV}[7,8,47]$ and the NLD calculations of Hilaire et al. [55] result in similar $T$ range for ${ }^{238} \mathrm{U}$. We checked $T$ in this range in the initial stage of our analysis and found that a value close to $T=0.3 \mathrm{MeV}$ is reasonable. Hence we fixed it and varied the parameter $k$ of the MGLO model and parameters of the SC peaks.

In addition, similar quality of agreement was achieved also with the MGLO model using excitation energy dependent $T$; see the Supplemental Material [35]. With this choice the MGLO model does not exactly follow the Brink hypothesis and the PSF shapes (for the decay from different excitation energies) are thus not necessarily similar to the constant- $T$ MGLO model. For different values of $k$ a good reproduction

\footnotetext{
${ }^{1}$ The corresponding adjustment of $E 2 \mathrm{PSF}$ is not a simple scaling.

${ }^{2}$ For $k=1.0$ the MGLO model is identical to the GLO model.
} 

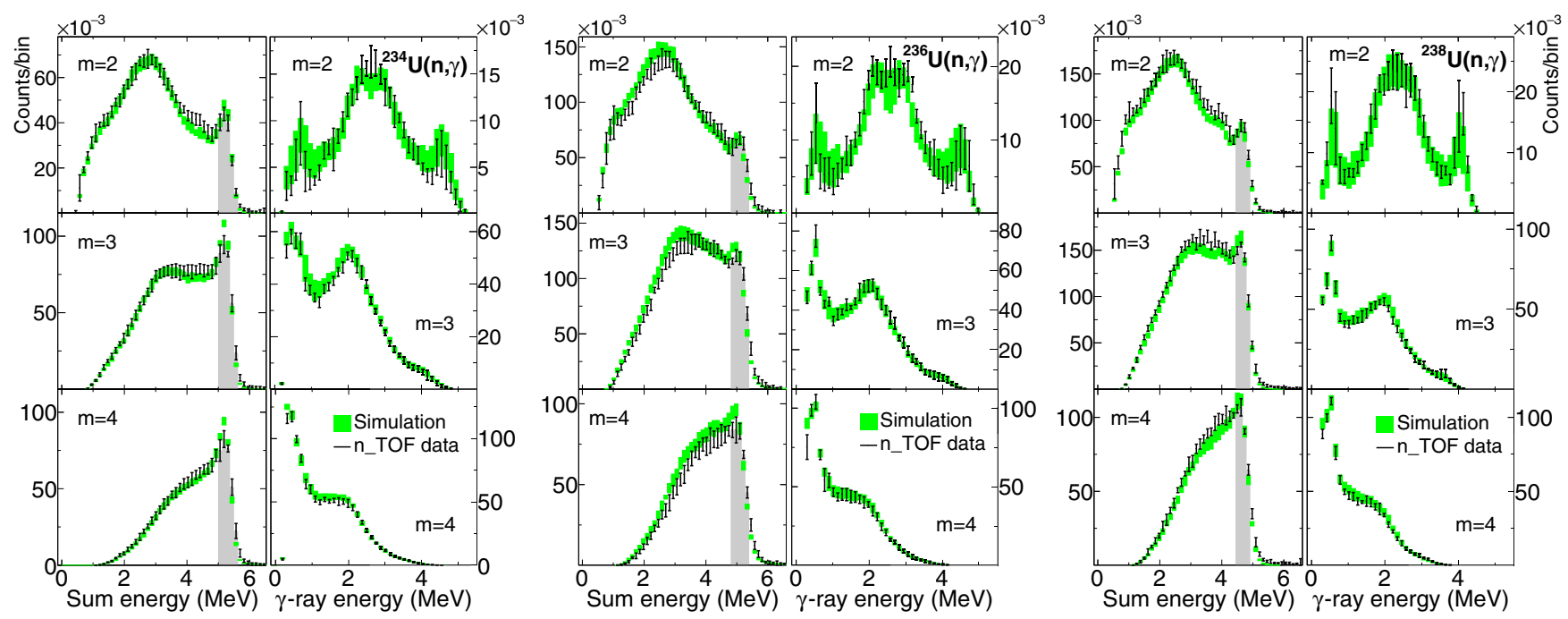

FIG. 9. Comparison of experimental and simulated sum-energy and MSC spectra for all three nuclei with the MGLO $(k=1.8$, $T=0.3 \mathrm{MeV}$ ) model. The label " $\gamma$-ray energy" refers to the deposited energy in any crystal.

of the experimental spectra is achieved with a different scissors mode shape; see Table I of the Supplemental Material [35]. With increasing $k$ the strength in the scissors mode region is shifted more to the higher-energy resonance. As a result, based on our data we are unable to decide if the actual $E 1$ PSF follows the exact form of the Brink hypothesis.

The results are virtually the same also with the CT NLD parametrization from Ref. [45]. In conjunction with the MGLO E1 model, the energy dependence of the NLD close to that given by the CT model gives satisfactory results. In reality, we performed a limited number of tests also with other $E 1$ models combined with BSFG and HFB [46] NLD models (mentioned in Sec. IV B); see the Supplemental Material [35]. We have never reached an agreement as good as that presented in this section. The tabulated HFB values are in principle subject to an additional energy-dependent normalization. We adopted the normalization available in TALYS 1.95 [56] and different normalizations have not been addressed in this work.

\section{Sensitivity of our data}

The absence of a good statistical criterion for a description of the agreement between simulations and experiment (together with the trial-and-error basis of the method) does not easily allow an accurate determination of parameter uncertainties. Despite this fact it should be stressed that the spectra, especially for $m=2$ and 3, are very sensitive to the positions of the SC peaks. The resonance energies are thus the same in all the model combinations yielding a good agreement with experimental data and are determined with an accuracy better than $0.1 \mathrm{MeV}$.

We performed tests in which we withdrew one or the other resonance of the double-resonance SC; see Fig. 10 and analogous figures in the Supplemental Material [35]. From the results we can conclude that the bump around $2 \mathrm{MeV}$ in $m=$ 3, 4 MSC spectra is significantly populated by the two-step cascades via the lower-energy SC followed by a (not necessarily statistical) low energy third (and fourth) step, while the middle part of $m=2$ MSC spectra also by two-step cascades via the higher-energy SC. This implies that the sensitivity to the parameters of the lower-energy SC is mostly given by the shape of $m=3,4 \mathrm{MSC}$ spectra, while the parameters of the higher-energy SC are mostly determined by the $m=2 \mathrm{MSC}$ spectra. We mention that, although a satisfactory description of the sum-energy spectra for ${ }^{235} \mathrm{U}$ (see the Supplemental Material [35]) was achieved when removing the higher-energy SC, the $m=2$ MSC spectrum clearly indicates that the energy makeup of the cascades contributing to the $m=2$ MSC spectrum is not correct. Hence the good match of the sum-energy

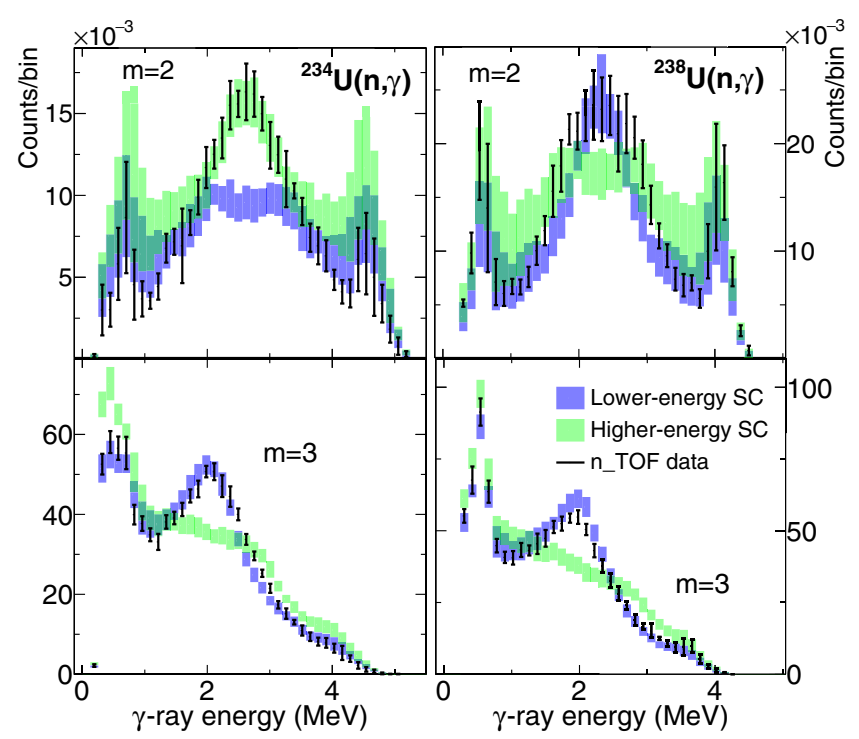

FIG. 10. Comparison of experimental MSC spectra for ${ }^{234} \mathrm{U}(n, \gamma)$ (left) and ${ }^{238} \mathrm{U}(n, \gamma)$ (right) with simulations in which one of the double-resonance SC was withdrawn. The simulations are labeled with the kept SC resonance. The $m=4$ MSC spectra are not shown as they exhibit the same behavior as the $m=3$ ones. The label " $\gamma$-ray energy" refers to the deposited energy in any crystal. 
spectra is purely accidental and indicates the need to use as many observables as possible to find a reasonable description of the $\gamma$ decay. The differences in predicted $m=2 \mathrm{MSC}$ spectra in three isotopes results solely from their different $S_{n}$. A simultaneous analysis of three isotopes (assuming the same or similar PSF models) thus significantly reduces a possibility of finding a false solutions coming from accidental agreement of simulations with experiment.

From the above, one could expect that a single-resonance $\mathrm{SC}$ would not lead to a satisfactory description of the experimental spectra. The simulations with a single-resonance SC provide good description only for ${ }^{235} \mathrm{U}$; see the Supplemental Material [35]. The reason is likely a favorable interplay between the value of $S_{n}$, the shape and the position of the maximum in the $m=2$ MSC spectrum, and the position of the bump at $\approx 2 \mathrm{MeV}$ in $m=3,4 \mathrm{MSC}$ spectra in this isotope. The triangle-like shape of $m=2$ MSC spectrum with the bumps in $m=3,4 \mathrm{MSC}$ spectra practically at $2 \mathrm{MeV}$ together with the highest $S_{n}$ of $5.3 \mathrm{MeV}$ allow for the use of single-resonance $\mathrm{SC}$ at $2.45 \mathrm{MeV}$ with the width of 1.25 $\mathrm{MeV}$ and maximum cross sections of $1.2 \mathrm{mb}$ for ${ }^{235} \mathrm{U}$. On the other hand, for the other two isotopes the plateau around the midpoint of the $m=2$ MSC spectra in combination with their $S_{n}$ 's requires a relatively wide $\mathrm{SC}$ at $\approx 2.4 \mathrm{MeV}$, which is unable to describe the relatively narrow bumps near 2 $\mathrm{MeV}$ in the $m=3,4 \mathrm{MSC}$ spectra. If the single resonance SC is tuned to $m=3,4 \mathrm{MSC}$ spectra, the resulting bumps in simulated $m=2 \mathrm{MSC}$ spectra are too narrow. Although changes in predictions with respect to double-resonance SC are not dramatic, our data favor the double-resonance SC. This conclusion is consistent with experimental findings of the Oslo group and theoretical explanations; see Refs. [8,57] and references therein.

We have to admit that although we attribute the needed resonances between 2 and $3 \mathrm{MeV}$ to the scissors mode, we can not unambiguously confirm their $M 1$ character. We performed tests with the $E 1$ character of these resonances and were able to reach a similar description of the experimental spectra; see the Supplemental Material [35]. However, in light of other findings, especially the NRF result of Hammond et al. [14] and the situation in deformed rare-earth nuclei, it is natural to assume that the needed strength corresponds to the scissors mode.

Guttormsen et al. [7] suggested a presence of pygmy resonance in the $E 1$ PSF with parameters mentioned in Table II. We have performed a few simulations for ${ }^{235} \mathrm{U}$, which should be the most sensitive because of the highest $S_{n}$, adding such pygmy resonance to the MGLO model of $E 1$ PSF and varying its maximum cross section. Our data exclude a presence of such pygmy resonance with $\sigma \gtrsim 2 \mathrm{mb}$.

\section{DISCUSSION OF RELATED OBSERVABLES}

\section{A. Total radiative width}

As mentioned above the sum-energy and MSC spectra are insensitive to the absolute scale of PSFs. Nonetheless, the scale impacts the total radiative width $\Gamma_{\gamma}$, the quantity related to the $\gamma$ decay that enters the cross section calculations.
TABLE III. Total radiative widths $\Gamma_{\gamma}$ of $s$-wave resonances obtained with different model combinations. The combinations labeled $\operatorname{MGLO}(k)$ consisted of the CT NLD model [50] and the MGLO $E 1 \mathrm{PSF}$ model with a constant temperature of $T=0.3 \mathrm{MeV}$ unless specified otherwise. Values for more model combinations can be found in the Supplemental Material [35].

\begin{tabular}{llll}
\hline \hline \multirow{2}{*}{ Model combination } & \multicolumn{3}{c}{$\Gamma_{\gamma}(\mathrm{meV})$} \\
\cline { 2 - 4 } PSF-LD & ${ }^{234} \mathrm{U}(n, \gamma)$ & ${ }^{236} \mathrm{U}(n, \gamma)$ & ${ }^{238} \mathrm{U}(n, \gamma)$ \\
\hline RIPL-3 & $16.1(2)$ & $12.9(2)$ & $9.5(2)$ \\
IAEA-19 & $29.4(6)$ & $19.3(5)$ & $13.9(5)$ \\
Oslo & $19.9(4)$ & $20.4(6)$ & $18.6(8)$ \\
DANCE & $22.0(5)$ & $17.2(4)$ & $15.9(6)$ \\
MGLO(1.8) & $25.4(7)$ & $20.1(5)$ & $15.9(6)$ \\
MGLO(2.5) & $30.5(10)$ & $23.9(7)$ & $18.8(7)$ \\
MGLO(3.0) & $39.0(12)$ & $30.9(9)$ & $24.3(9)$ \\
MGLO( $k, T(E))$ & $26.7(7)^{\mathrm{a}}$ & $24.5(6)^{\mathrm{b}}$ & $19.2(7)^{\mathrm{c}}$ \\
Mughabghab's atlas [58] & $25.3(10)$ & $23.4(8)$ & $23.36(31)$ \\
Mughabghab's atlas [51] & $36.7(7)$ & $23.4(8)$ & $22.9(4)$ \\
JEFF-3.3 [5,59] & 26.0 & 23.0 & 22.5 \\
ENDF/B-VIII.0 [4] & 26.0 & 19.5 & 22.5 \\
\hline \hline
\end{tabular}

${ }^{\mathrm{a} W i t h} k=1.8$.

${ }^{\mathrm{b}}$ With $k=2.5$.

${ }^{\mathrm{c}}$ With $k=3.0$.

The $\Gamma_{\gamma}$ can be obtained from DICEBOX calculations and are for a few model combinations compared to the literature in Table III for $s$-wave resonances; values for more model combinations can be found in the Supplemental Material [35]. The uncertainty in simulations represents the standard deviation of $\Gamma_{\gamma}$ from different artificial nuclei as calculated by DICEBOX.

The simulated $\Gamma_{\gamma}$ show a significant change with the adopted NLD model (see the Supplemental Material [35]); the model combinations labeled $\operatorname{MGLO}(k)$ presented in Table III used the CT NLD of Ref. [50]. This change is not only due to a different energy dependence of the adopted NLD modelscombinations of fixed PSFs with BSFG models yield about $2 \times$ higher $\Gamma_{\gamma}$ compared to the CT ones-but also due to different parametrizations of the given NLD model. Namely, the $\Gamma_{\gamma}$ with the CT model parametrization from Ref. [45] are higher than those listed in Table III by about $5-7 \%$ for ${ }^{234,236} \mathrm{U}$ and by about $25 \%$ for ${ }^{238} \mathrm{U}$ resonances virtually independent of adopted PSF models.

Although the general agreement between simulated and evaluated $\Gamma_{\gamma}$ values is relatively good, within about $30 \%$ for majority of model combinations, none of them is able to reproduce the evaluated values within listed uncertainties for all three isotopes. According to Ref. [58], the experimental $\Gamma_{\gamma}$ have similar values for all three isotopes. It shows that the CT NLD model with fixed parameters in conjunction with fixed PSFs yields very similar values of $\Gamma_{\gamma}$ for a broad range of initial excitation energies, which is a consequence of the NLD functional form. This indicates that the experimental similarity of $\Gamma_{\gamma}$ for all three nuclei can be reached for the same PSFs only if the NLD is almost identical in all three nuclei. The experimental data on the resonance spacing [51] as well as the deduced NLD parameters [50], however, indicate a similar, albeit different NLD. The fact that different $k$-specifically, 
$k \approx 1.8,2.5$, and 3.0 - in conjunction with different $\mathrm{SC}$ are needed to reproduce the compiled values [58] of $\Gamma_{\gamma}$ in ${ }^{235} \mathrm{U}$, ${ }^{237} \mathrm{U}$, and ${ }^{239} \mathrm{U}$ respectively, is thus a consequence of different NLDs in three isotopes. The change of PSFs thus could have been expected from a combination of experimental $\Gamma_{\gamma}$ values for all three isotopes, their resonance spacing, and data on low-lying levels. We consider the best resulting model combination for each isotope the one which well reproduces the experimental spectra as well as the value of $\Gamma_{\gamma}$.

In any case, we would like to stress that a significant contribution to $\Gamma_{\gamma}$ comes from the scissors mode, which is responsible for about a half of $\Gamma_{\gamma}$ for the model combinations considered in this work that nicely reproduce the experimental spectra.

\section{B. Scissors mode parameters}

The discussion above strongly indicates that the actual strength of the scissors mode depends on the isotope. Our required scissors mode strength is in any case much higher than that in all tested model combinations from the literature; see Fig. 6. Specifically, the integrated SC strength corresponding to the model combination using the MGLO E1 model with $k=1.8$ and 3.0 is about 17 and $30 \mu_{N}^{2}$, respectively. For reference, the integrated SC strength is $\approx 9 \mu_{N}^{2}$ for all three uranium isotopes analyzed with the Oslo method [7] and the DANCE analysis [11] provided a lower limit of $\approx 11 \mu_{N}^{2}$.

The SC strength could be compared also to results reported from the NRF experiments. Yevetska et al. [13] deduced $M 1$ strength of $3.6(13) \mu_{N}^{2}$ in the energy range of $1.6-2.8 \mathrm{MeV}$ in ${ }^{235} \mathrm{U}$, our corresponding value is $\approx 12 \mu_{N}^{2}$. However, only seven transitions between 1.6 and $2.1 \mathrm{MeV}$ were observed in Ref. [13], while the CT NLD model predicts some 150 levels; a significant fraction of the strength thus remains unseen in the NRF experiment. The situation is different in even-even nuclei, where the NRF should observe a significant portion of the strength in the range below about $4 \mathrm{MeV}$. Specifically, the observed strength between 2 and $3.5 \mathrm{MeV}$ in ${ }^{238} \mathrm{U}$ was $8(1) \mu_{N}^{2}$ [14]. This value should be considered as a lower limit for the actual strength. The predicted strength in the same energy range with the Oslo [7], DANCE [11], MGLO(1.8), and MGLO(3.0) model combinations is about 5, 7, 11, and $18 \mu_{N}^{2}$. Assuming the SC is not significantly stronger in an even-even isotope when compared to neighboring odd ones, these values indicate that the SC strength from the literature $[7,11]$ was underestimated.

\footnotetext{
${ }^{3}$ Ullmann et al. did not explicitly state that their value is a lower limit, but such a conclusion is evident from the comparison of simulated and experimental MSC spectra in Fig. 4 and the total radiative width in Table III of Ref. [11]
}

\section{CONCLUSIONS}

The total absorption calorimeter at the $\mathrm{n}$-TOF facility (CERN) was used to measure the $\gamma$-ray cascades following neutron capture on the nuclei ${ }^{234} \mathrm{U},{ }^{236} \mathrm{U}$, and ${ }^{238} \mathrm{U}$. The background-corrected sum-energy and multistep cascade spectra were constructed from data corresponding to the decay of 6-8 well-isolated $s$-wave neutron resonances below energy of about $140 \mathrm{eV}$. These spectra were then compared to statistical model predictions coming from different models of photon strength functions and nuclear level density. The simulated spectra were obtained by applying the GEANT 4 simulations of the TAC response to $\gamma$-ray cascades produced by the DICEBOX code. The MSC spectra from n_TOF were extensively exploited for getting information on PSFs and NLD for the first time at this level of detail. In addition the analysis included external constraints to preserve the agreement with existing photoabsorption data and with the total radiative width.

We found that none of the model combinations available in the literature can precisely reproduce our experimental spectra for all three nuclei. We further performed an extensive search for a model combination common to all three isotopes that would reproduce our spectra. We found a group of model combinations that nicely describes them. These combinations require a nuclear level density with an energy dependence close to the constant temperature model and a strong influence of the scissors mode, which definitely has a more complex structure than a single Lorentzian. The mode is likely double resonance and relatively wide, strongly influencing the decay via $\gamma$ rays with energy between 1.5 and $3.5 \mathrm{MeV}$. The SC has very likely a strength higher than about $17 \mu_{N}^{2}$ and is responsible for about half of the total radiative width of neutron resonances. Our calculations show that this contribution to the radiative width increases the neutron capture cross section by about $50 \%$, which is in accord with calculations by Ullmann et al. [11].

While in this work we have largely focused on analytical NLD and PSF models, we also included some models based on recent progress on microscopic calculations. Fine-tuning the energy-dependent normalizations associated with the microscopic NLD models is, however, outside the scope of this paper. The present work has shown the comparison of newly extracted experimental observables from n_TOF, sensitive to the nuclear level density and the dipole $\gamma$-ray strength, with extensive model calculations. The employed methodology may trigger future work including a reanalysis of other, previously taken data sets, as well as new measurements at $\mathrm{n}_{-} \mathrm{TOF}$ with the total absorption calorimeter using nuclei in different mass ranges.

\section{ACKNOWLEDGMENTS}

The work was supported by the MŠMT of the Czech Republic, the Charles University Project No. UNCE/SCI/013, and by the funding agencies of the participating institutes.
[1] W. Hauser and H. Feshbach, Phys. Rev. 87, 366 (1952).
[2] M. Arnould and S. Goriely, Prog. Part. Nucl. Phys. 112, 103766 (2020). 
[3] J. J. Cowan, C. Sneden, J. E. Lawler, A. Aprahamian, M. Wiescher, K. Langanke, G. Martínez-Pinedo, and F.-K. Thielemann, Rev. Mod. Phys. 93, 015002 (2021).

[4] D. A. Brown et al., Nucl. Data Sheets 148, 1 (2018), Special Issue on Nuclear Reaction Data.

[5] A. J. M. Plompen et al., Eur. Phys. J. A 56, 181 (2020).

[6] I. Dillmann, M. Heil, F. Käppeler, R. Plag, T. Rauschert, and F. Thielemann, AIP Conf. Proc. 819, 123 (2006).

[7] M. Guttormsen, L. A. Bernstein, A. Görgen, B. Jurado, S. Siem, M. Aiche, Q. Ducasse, F. Giacoppo, F. Gunsing, T. W. Hagen, A. C. Larsen, M. Lebois, B. Leniau, T. Renstrøm, S. J. Rose, T. G. Tornyi, G. M. Tveten, M. Wiedeking, and J. N. Wilson, Phys. Rev. C 89, 014302 (2014).

[8] T. A. Laplace, F. Zeiser, M. Guttormsen, A. C. Larsen, D. L. Bleuel, L. A. Bernstein, B. L. Goldblum, S. Siem, F. L. Bello Garrote, J. A. Brown, L. C. Campo, T. K. Eriksen, F. Giacoppo, A. Görgen, K. Hadyńska-Klçk, R. A. Henderson, M. Klintefjord, M. Lebois, T. Renstrøm, S. J. Rose et al., Phys. Rev. C 93, 014323 (2016).

[9] F. Zeiser, G. M. Tveten, G. Potel, A. C. Larsen, M. Guttormsen, T. A. Laplace, S. Siem, D. L. Bleuel, B. L. Goldblum, L. A. Bernstein, F. L. Bello Garrote, L. Crespo Campo, T. K. Eriksen, A. Gorgen, K. Hadynska-Klek, V. W. Ingeberg, J. E. Midtbo, E. Sahin, T. Tornyi, A. Voinov, M. Wiedeking, and J. Wilson, Phys. Rev. C 100, 024305 (2019).

[10] J. L. Ullmann, T. Kawano, T. A. Bredeweg, A. Couture, R. C. Haight, M. Jandel, J. M. O'Donnell, R. S. Rundberg, D. J. Vieira, J. B. Wilhelmy, J. A. Becker, A. Chyzh, C. Y. Wu, B. Baramsai, G. E. Mitchell, and M. Krtička, Phys. Rev. C 89, 034603 (2014).

[11] J. L. Ullmann, T. Kawano, B. Baramsai, T. A. Bredeweg, A. Couture, R. C. Haight, M. Jandel, J. M. O’Donnell, R. S. Rundberg, D. J. Vieira, J. B. Wilhelmy, M. Krtička, J. A. Becker, A. Chyzh, C. Y. Wu, and G. E. Mitchell, Phys. Rev. C 96, 024627 (2017), Erratum in Ref. [60].

[12] J. Kopecky, S. Goriely, S. Péru, S. Hilaire, and M. Martini, Phys. Rev. C 95, 054317 (2017).

[13] O. Yevetska, J. Enders, M. Fritzsche, P. von Neumann-Cosel, S. Oberstedt, A. Richter, C. Romig, D. Savran, and K. Sonnabend, Phys. Rev. C 81, 044309 (2010).

[14] S. L. Hammond, A. S. Adekola, C. T. Angell, H. J. Karwowski, E. Kwan, G. Rusev, A. P. Tonchev, W. Tornow, C. R. Howell, and J. H. Kelley, Phys. Rev. C 85, 044302 (2012).

[15] R. R. Hilton, in Proceedings of the International Conference on Nuclear Structure, Dubna, USSR, 1976, edited by V. G. Soloviev (JINR, Dubna, 1976).

[16] N. Lo Iudice and F. Palumbo, Phys. Rev. Lett. 41, 1532 (1978).

[17] D. Bohle, A. Richter, W. Steffen, A. Dieperink, N. Lo Iudice, F. Palumbo, and O. Scholten, Phys. Lett. B 137, 27 (1984).

[18] U. Berg, C. Bläsing, J. Drexler, R. Heil, U. Kneissel, W. Naatz, R. Ratzek, S. Schennach, R. Stock, T. Weber, H. Wickert, B. Fischer, H. Hollick, and D. Kollewe, Phys. Lett. B 149, 59 (1984).

[19] M. Krtička, F. Bečváŕ, J. Honzátko, I. Tomandl, M. Heil, F. Käppeler, R. Reifarth, F. Voss, and K. Wisshak, Phys. Rev. Lett. 92, 172501 (2004).

[20] S. Goriely, P. Dimitriou, M. Wiedeking, T. Belgya, R. Firestone, J. Kopecky, M. Krtička, V. Plujko, R. Schwengner, S. Siem, H. Utsunomiya, S. Hilaire, S. Péru, Y. S. Cho, D. M. Filipescu, N. Iwamoto, T. Kawano, V. Varlamov, and R. Xu, Eur. Phys. J. A 55, 172 (2019).
[21] R. Capote, M. Herman, P. Obložinský, P. G. Young, S. Goriely, T. Belgya, A. V. Ignatyuk, A. J. Koning, S. Hilaire, V. A. Plujko, M. Avrigeanu, O. Bersillon, M. B. Chadwick, T. Fukahori, Z. Ge, Y. Han, S. Kailas, J. Kopecky, V. M. Maslov, G. Reffo et al., Nucl. Data Sheets 110, 3107 (2009).

[22] W. Dridi, Mesure de la section efficace de capture neutronique de l' ${ }^{234} \mathrm{U}$ à n_TOF au CERN pour les réacteurs nucléaires de Génération IV (in French), Ph.D. thesis, Université d'Evry Val d'Essonne, 2006, https://cds.cern.ch/record/1059274.

[23] C. Lampoudis et al. (n_TOF Collaboration), in ND2007, International Conference on Nuclear Data for Science and Technology 2007 (EDP Sciences, Les Ulis, France, 2007).

[24] M. J. Vermeulen, Measurement of the ${ }^{236} \mathrm{U}(n, \gamma)$ cross section for the thorium fuel cycle at the CERN n_TOF facility, Ph.D. thesis, University of York, 2015, http://etheses.whiterose.ac.uk/ 9681.

[25] M. Mastromarco et al. (n_TOF Collaboration), EPJ Web Conf. 146, 11054 (2017).

[26] T. Wright, High Accuracy Measurement of the ${ }^{238} \mathrm{U}(n, \gamma)$ Cross Section at the CERN n_TOF Facility, Ph.D. thesis, University of Manchester 2014, https://cds.cern.ch/record/1976152.

[27] T. Wright et al. (n_TOF Collaboration), Phys. Rev. C 96, 064601 (2017).

[28] J. Moreno-Soto et al., EPJ Web Conf. 211, 02002 (2019).

[29] J. Moreno Soto, Study of the photon strength functions and level densities in the gamma decay following neutron capture on the isotopes ${ }^{234} \mathrm{U},{ }^{236} \mathrm{U}$, and ${ }^{238} \mathrm{U}$, Ph.D. thesis, University ParisSaclay, 2020, https://cds.cern.ch/record/2745718.

[30] C. Borcea, P. Cennini, M. Dahlfors, A. Ferrari, G. GarciaMuñoz, P. Haefner, A. Herrera-Martínez, Y. Kadi, V. Lacoste, E. Radermacher, F. Saldaña, V. Vlachoudis, L. Zanini, C. Rubbia, S. Buono, V. Dangendorf, R. Nolte, and M. Weierganz, Nucl. Instrum. Methods Phys. Res., Sect. A 513, 524 (2003).

[31] C. Guerrero et al. (n_TOF Collaboration), Eur. Phys. J. A 49, 27 (2013).

[32] C. Guerrero et al., Nucl. Instrum. Methods Phys. Res., Sect. A 608, 424 (2009).

[33] E. Berthoumieux et al. (n_TOF Collaboration), Internal report, 2004 (unpublished).

[34] E. Mendoza et al. (n_TOF Collaboration), Nucl. Instrum. Methods Phys. Res., Sect. A 768, 55 (2014).

[35] See Supplemental Material at http://link.aps.org/supplemental/ 10.1103/PhysRevC.105.024618 for additional figures.

[36] C. E. Porter and R. G. Thomas, Phys. Rev. 104, 483 (1956).

[37] S. Valenta, B. Baramsai, T. A. Bredeweg, A. Couture, A. Chyzh, M. Jandel, J. Kroll, M. Krticka, G. E. Mitchell, J. M. ODonnell, G. Rusev, J. L. Ullmann, and C. L. Walker, Phys. Rev. C 96, 054315 (2017).

[38] F. Bečvář, Nucl. Instrum. Methods Phys. Res., Sect. A 417, 434 (1998), Erratum in Ref. [61]; M. Krtička and S. Valenta, https: //www-nds.iaea.org/dicebox/, 2018.

[39] E. Browne and J. K. Tuli, Nucl. Data Sheets 122, 205 (2014).

[40] M. S. Basunia, Nucl. Data Sheets 107, 2323 (2006).

[41] E. Browne and J. K. Tuli, Nucl. Data Sheets 122, 293 (2014).

[42] S. Agostinelli et al., Nucl. Instrum. Methods Phys. Res., Sect. A 506, 250 (2003).

[43] C. Guerrero et al. (n_TOF Collaboration), Nucl. Instrum. Methods Phys. Res., Sect. A 671, 108 (2012).

[44] J. Kopecky, M. Uhl, and R. E. Chrien, Phys. Rev. C 47, 312 (1993). 
[45] T. von Egidy and D. Bucurescu, Phys. Rev. C 72, 044311 (2005), Erratum in Ref. [62].

[46] S. Goriely, S. Hilaire, and A. J. Koning, Phys. Rev. C 78, 064307 (2008).

[47] M. Guttormsen, B. Jurado, J. N. Wilson, M. Aiche, L. A. Bernstein, Q. Ducasse, F. Giacoppo, A. Görgen, F. Gunsing, T. W. Hagen, A. C. Larsen, M. Lebois, B. Leniau, T. Renstrøm, S. J. Rose, S. Siem, T. Tornyi, G. M. Tveten, and M. Wiedeking, Phys. Rev. C 88, 024307 (2013).

[48] M. Krtička, S. Goriely, S. Hilaire, S. Péru, and S. Valenta, Phys. Rev. C 99, 044308 (2019).

[49] D. M. Brink, Some aspect of the interaction of fields with matter, Ph.D. thesis, Oxford University (1955).

[50] T. von Egidy and D. Bucurescu, Phys. Rev. C 80, 054310 (2009).

[51] S. F. Mughabghab, Atlas of Neutron Resonances, Volume 2: Resonance Properties and Thermal Cross Sections $Z=61-102$ (Elsevier, Amsterdam, 2018).

[52] J. Kroll, B. Baramsai, G. E. Mitchell, U. Agvaanluvsan, F. Bečváŕ, T. A. Bredeweg, A. Chyzh, A. Couture, D. Dashdorj, R. C. Haight, M. Jandel, A. L. Keksis, M. Krtička, J. M. O'Donnell, W. Parker, R. S. Rundberg, J. L. Ullmann, S. Valenta, D. J. Vieira, C. Walker et al., Phys. Rev. C 88, 034317 (2013).
[53] J. T. Caldwell, E. J. Dowdy, B. L. Berman, R. A. Alvarez, and P. Meyer, Phys. Rev. C 21, 1215 (1980).

[54] M. B. Chadwick et al., Handbook for calculations of nuclear reaction data, Technical Report IAEA-TECDOC-1034 (International Atomic Energy Agency, Vienna, Austria, 1998).

[55] S. Hilaire, M. Girod, S. Goriely, and A. J. Koning, Phys. Rev. C 86, 064317 (2012).

[56] A. Koning and D. Rochman, Nucl. Data Sheets 113, 2841 (2012).

[57] M. Guttormsen, L. A. Bernstein, A. Bürger, A. Görgen, F. Gunsing, T. W. Hagen, A. C. Larsen, T. Renstrøm, S. Siem, M. Wiedeking, and J. N. Wilson, Phys. Rev. Lett. 109, 162503 (2012)

[58] S. F. Mughabghab, Atlas of Neutron Resonances (Elsevier, Amsterdam, 2006).

[59] A. J. Koning et al., J. Korean Phys. Soc. 59, 1057 (2011).

[60] J. L. Ullmann, T. Kawano, B. Baramsai, T. A. Bredeweg, A. Couture, R. C. Haight, M. Jandel, J. M. O’Donnell, R. S. Rundberg, D. J. Vieira, J. B. Wilhelmy, M. Krtička, J. A. Becker, A. Chyzh, C. Y. Wu, and G. E. Mitchell, Phys. Rev. C 104, 019902(E) (2021).

[61] F. Bečvář, Nucl. Instrum. Methods Phys. Res., Sect. A 935, 240 (2019).

[62] T. von Egidy and D. Bucurescu, Phys. Rev. C 73, 049901(E) (2006). 